Theme: Solidification / Casting

\title{
DYNAPHASE: ONLINE CALCULATION OF THERMODYNAMIC PROPERTIES DURING CONTINUOUS CASTING*
}

\begin{abstract}
Susanne Hahn ${ }^{1}$ Thomas Schaden ${ }^{2}$

\section{Abstract}

The Siemens VAI software model DynaPhase calculates thermodynamic properties depending on the chemical analysis of steel. It can be used online together with Siemens VAI's secondary cooling model SIMETAL Dynacs 3D which is a unique feature. Offline it can be used to investigate the thermodynamic properties of steel grades and therefore supports the development of new grades. The core of DynaPhase is a substitutional solution model for the Gibbs free energy to calculate multi-component phase diagrams. The parameters of this model are determined by employing the CALPHAD approach. This model is combined with a microsegregation model to account for the interdendritic solidification process. New DSC (differential scanning calometry) measurements of the department of metallurgy of the Mining University of Leoben have been used to improve the DynaPhase database for high Al or Si concentrations. By comparing this experimental data to calculations it has been found that some of the widely used commercial software tools do not describe the ternary system FeAIC for high aluminum concentration correctly. In this paper we will show how thermal properties of high Al grades change with the optimized database and what impact this has on the secondary cooling of continuous casting, e.g. how the solidus point shifts on the strand. Moreover we will show that the online use of DynaPhase has many advantages for the production. Since DynaPhase uses the actual chemical analysis of the steel grade and not just concentration ranges or averaged concentrations it is possible to optimize the cooling strategy during casting for the actual chemical analysis of the actual heat.
\end{abstract}

Keywords: Thermodynamics; Simulation; Automation; Continuous casting.

1 Dr. rer. nat., Researcher, Continuous Casting, Siemens VAl Metals Technologies GmbH, Linz, Austria.

2 Dr. Dipl.-Ing., Researcher, Continuous Casting, Siemens VAl Metals Technologies GmbH, Linz, Austria.

* Technical contribution to the $45^{\text {th }}$ Steelmaking Seminar, May $25^{\text {th }}-28^{\text {th }}, 2014$, Porto Alegre, RS, Brazil. 


\section{INTRODUCTION}

In the past, thermo mechanical strand treatment was a subject of the rolling process. Historically, continuous casting focused on controlling the strand surface temperature by secondary cooling [1,2] to prevent surface defects. In a next step temperature control was extended to account for the second ductility trough caused by phase transitions and precipitation in the solid state. It is of great importance to consider this effect during unbending of the strand and in hot direct charging processes $[3,4]$. With the introduction of dynamically adjustable segments it became possible to control the roll gap during casting [5]. With this innovation dynamic soft reduction could be introduced on an industrial scale [6,7]. Nowadays thermo mechanical processing is a standard feature of continuous casting technology. Employing metallurgical treatments make it possible to influence the as-cast grain structure, to improve surface quality and product shape and to reduce segregation and porosity. Metallurgical treatments are also applicable to make the strand more suitable for hot charging.

The modern continuous caster has many features and therefore great potential to actively influence the as-cast strand properties. This could reduce the energetically costly and elaborate strand treatment after the initial solidification in the caster. Therefore it also could help to reduce the $\mathrm{CO}_{2}$ emission of steel production.

In order to exploit the full potential of a state of the art continuous caster the underlying physical and chemical processes have to be mapped onto detailed process models. These models must be capable to accurately predict and control the process with respect to heat transfer in the strand, compound formation and phase transformations during cooling.

Simetal Dynacs 3D is a secondary cooling model system for continuous casting [8-10]. It consists of two modules. One module, the Thermal Tracker 3D solves the three dimensional heat transfer equation for the entire strand and predicts the temperature distribution of the strand during casting. The second model is the Setpoint Calculator which controls the secondary cooling loops. It regulates the water flow, the air pressure and the position of the spray nozzles to meet some predefined temperature profile of the strand. Solving the three dimensional heat transfer equation is a mathematical problem. Many authors have addressed this problem, but all these algorithms have in common that they either assume constant density or do not conserve energy and mass [11-13]. Unlike other models, the Simetal Dynacs 3D model considers the temperature-dependent density and globally conserves mass and enthalpy. With modern computers the resulting equation can be handled numerically with great accuracy and efficiency. However, in order to do so steel grade specific material properties like liquidus and solid temperature have to be provided.

An additional software package, DynaPhase, calculates all the thermo-physical data needed by Simetal Dynacs 3D. DynaPhase is available as an online tool which calculates liquidus and solidus temperature and material properties of the actual steel grade analysis. This is a unique feature and makes it stand out from the competition. Simetal Dynacs 3D will then use this data instead of data generated for grade groups with a certain range for the concentration of the chemical analysis. Thus the prediction of quantities as solidification point in the caster and the temperature distribution of the strand during casting will be more accurate and therefore allows for precise metallurgical treatment which leads to an enhanced quality of the products.

* Technical contribution to the $45^{\text {th }}$ Steelmaking Seminar, May $25^{\text {th }}-28^{\text {th }}, 2014$, Porto Alegre, RS, Brazil. 
In this paper we will focus on the software package DynaPhase. We will start with a brief overview of its models and the used thermo dynamical database. After that we will show at two examples how important accurate calculation results are for a beneficial utilization of metallurgical treatments. Finally we will discuss how DynaPhase can help to increase the productivity of the caster and the quality of the products.

\section{MODEL AND DATABASE}

DynaPhase is a software package which simulates the phase transitions and the microsegregation under the cooling condition in a continuous caster. Moreover it calculates the temperature depending material data needed by Simetal Dynacs 3D. It is available as an off line and an online version. The off line version allows to precalculate material properties for an arbitrary steel grade analysis within the scope of the database. As online version it takes the chemical analysis of the actual steel grade and calculates the material properties for Simetal Dynacs 3D just before start casting. DynaPhase consists of two main parts. The first part describes the solidification of the liquid steel in the high temperature range, the second part deals with the solid-solid phase transitions in the lower temperature range.

Part one, the high temperature range, is based on two main models. The first model is a substitutional solution model with Redlich-Kister polynomials for the excess term of the Gibbs free energy and describes the thermodynamic equilibrium [14-16]. This model has many free parameter which have to be determined by deploying the CALPHAD approach [17]. To simulate solidification during continuous casting it is very important to cover segregation phenomena correctly. Thus the second model describes interdendritic solidification depending on external cooling rates. Therefore a representative volume element located in the mushy zone is considered. At the interface between different phases mass balance is requested. The diffusion of the solutes in the different phases obeys Fick's second law. The two models are then combined by assuming thermodynamic equilibrium at the phase interfaces. The resulting system of equations is solved numerically with Newton's method.

Part two, the low temperature range; is described by a kinetic reaction model developed by Johnson-Mehl and by Avrami and a FeC phase diagram which is shifted by the concentration of the alloying elements. The free parameters of the model have been determined with help of experiments. With this model the solidsolid phase transformation from austenite to ferrite, pearlite, bainite and martensite are calculated, depending on the actual steel grade analysis and the cooling curve.

As output DynaPhase calculates liquidus and solid temperature, phase fraction depending on temperature and temperature depending material properties as density and thermal conductivity.

It is well known that the formation of precipitations may influence quantities like the solidus temperature. Hence DynaPhase accounts for non-metallic precipitations by an extension of the model. Therefore, for example, the enrichment of sulfur in the liquid phase during solidification, which is typically retained by manganese by forming manganese sulfide (MnS), is expressed more correctly by the extended model. So far the model includes precipitation of sulfides as MnS, nitrides as TiN and carbides as TiC.

The benefit of the coupled models in DynaPhase is the ability to characterize the complete solidification process. It describes the transformation from the liquid phase into ferrite or austenite as well as the three-phase region where liquid is transformed

* Technical contribution to the $45^{\text {th }}$ Steelmaking Seminar, May $25^{\text {th }}-28^{\text {th }}, 2014$, Porto Alegre, RS, Brazil. 
into austenite and the transformation of previously built ferrite into austenite has already started. This means that all energetically possible phases in the peritectic region are properly accounted for when the solidification interval is determined.

\subsection{Database Extension: the CALPHAD Approach}

A crucial point for a successful calculation is the quality of the thermodynamic database. The many free parameters of the Gibbs energy model have to be determined with help of the CALPHAD approach. In a first step the database has been built by collecting parameter sets published in the relevant literature over the last forty years. Since we are only interested in continuously cast steel, we could restrict our search to the iron rich corner of the phase diagram with limitations for the concentrations of the other elements. It has been given proof in comparison with experimental measurements and with other commercial thermodynamic software tools as well as in practical applications on continuous casting machines, that the resulting database covers carbon steel grades and also stainless steel grades quite well. However the database is only valid within certain concentration ranges for the alloying elements which are sufficient for most of the common steel grades.

On the other hand, our customers are constantly pushing the limits by designing new steel grades with special material properties. The development of TRIP steel grades with high $\mathrm{Al}$ and rather high Si concentration is one example of this trend. These new grades have been beyond the scope of the DynaPhase database. Inspecting the literature we could not find experimental data to extend the database in this direction. Thus we started a research project together with voestalpine stahl, Linz and the department of metallurgy of the Mining University of Leoben. In this project high precision DSC (differential scanning calometry) measurements to determine the equilibrium phase transitions have been conducted $[18,19]$. So far the ternary systems FeSiC and FeAIC with Silicon concentration up to $3.3 \mathrm{wt} \%$ and Al concentration of up to $3.4 \mathrm{wt} \%$ have been investigated systematically. It was possible to experimentally determine pseudo binary cuts of FeAIC phase diagrams. Comparison of these cuts to corresponding phase diagrams calculated with FactSage [20] (database SGTE2007) and Thermo-Calc [21] (database TCFE6) has shown that the commercial software packages are not able to reproduce the high $\mathrm{Al}$ measurements always correctly. Both programs reproduce the liquidus line very nicely but greater discrepancies between calculation and measurement have been found for the other phase transformation lines. For some high Al samples FactSage does not even predicted the right phase transformation. For samples with other concentrations we sometimes found deviations from the experimental transformation temperatures of up to $50^{\circ} \mathrm{C}$ for FactSage and of over $30^{\circ} \mathrm{C}$ for Thermo-Calc. We would like to stress that this differences have only be found for high $\mathrm{Al}$ concentrations. For lower Al concentration the correspondence between experiment and calculation was good for FactSage as well as for Thermo-Calc. We are aware of the fact that there are more recent versions of both databases available which possibly contain improved parameter sets for the FeAIC system for high Al concentrations.

We were using these DSC measurements to tune and extend the DynaPhase database for high $\mathrm{Al}$ and high Si content (with the restriction that concentrations of other alloying elements are not extremely high). DynaPhase is now valid for Si and Al concentrations up to 3.5 wt\%. Further DSC measurements are underway and will be used to further improve and extend the DynaPhase database.

* Technical contribution to the $45^{\text {th }}$ Steelmaking Seminar, May $25^{\text {th }}-28^{\text {th }}, 2014$, Porto Alegre, RS, Brazil. 


\section{RESULTS AND DISCUSSION}

To show how the changes of the database are reflected in Simetal Dynacs 3D we choose a simple example steel grade with 0.22 wt $\%$ carbon and 1.97 wt $\%$ aluminum, which we call Al197. For the old DynaPhase database grades with $1.97 \mathrm{wt} \% \mathrm{Al}$ are well outside the range of validity. Only grades with Al up to $0.5 \mathrm{wt} \%$ are allowed for the old database. Ignoring this fact we determined the material properties with DynaPhase assuming an average cooling rate of $0.5^{\circ} \mathrm{C} / \mathrm{s}$ with the old database and the new optimized database. In Figure 1 we plot the resulting solid fraction against the temperature. The old database calculates the liquidus temperature correctly, but for the solidus point we find a discrepancy from almost $40^{\circ} \mathrm{C}$ between the two simulations. But what would this mean for continuous casting when Simetal Dynacs 3D controls the spray water of the secondary cooling loops?

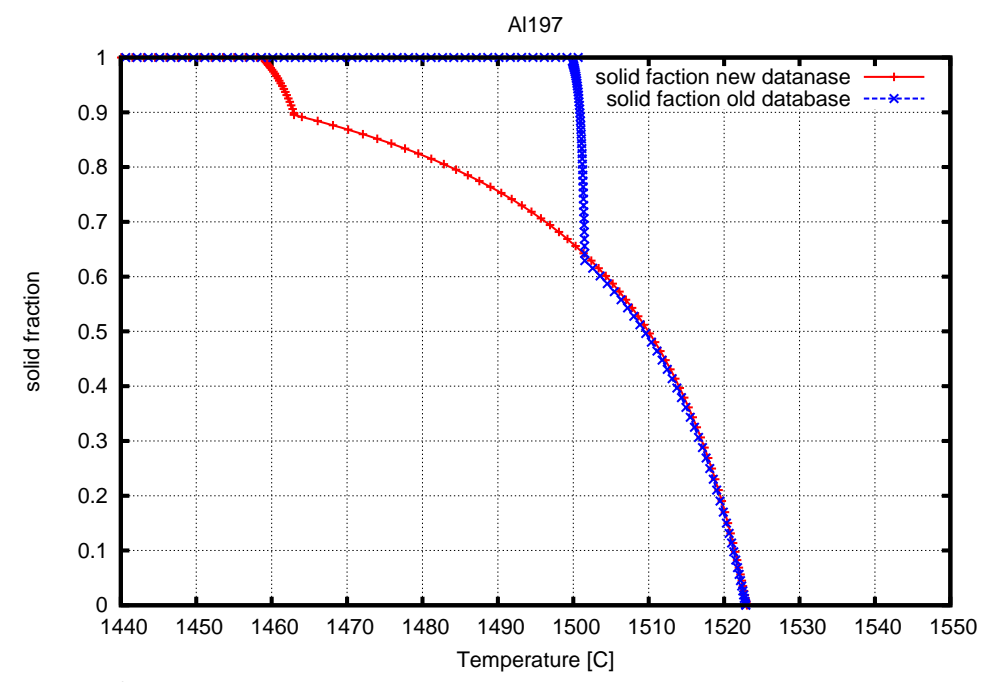

Figure 1. Solid fraction for the grade Al197 calculated with the DynaPhase database before the optimization (blue) and after the optimization (red). The liquidus temperature is the same, but the solidus temperature is for the red curve $40^{\circ} \mathrm{C}$ lower than for the blue curve.

To investigate this we run two Simetal Dynacs 3D simulations for a standard slab caster format $225 \times 1500 \mathrm{~mm}^{2}$ and a medium cooling practice. In Figure 2 we show the liquidus and solidus isotherms on the narrow face of the strand at a cut through the center of the slab. Thereby the width position $0 \mathrm{~m}$ represents the narrow face surface of the strand and the width position $0.1125 \mathrm{~m}$ the center of the strand. The red curves represent the results with the material properties calculated with the old database whereas the results of the simulation with the new database are given in blue. Since the liquidus temperatures for both sets of material properties are almost identical we find no difference of the liquidus isotherm on the strand. A shift of $40^{\circ} \mathrm{C}$ for the solidus temperature leads to a shift of the point of complete solidification of almost $1.7 \mathrm{~m}$ on the strand. Such a finding could severely influence the performance of strand treatment techniques like soft reduction. For beneficial use of soft reduction it is essential to apply the reduction at the right position at the right solid fraction. If we apply soft reduction too early for a too low solid fraction this could lead to crack formation but on the other hand if we apply soft reduction too late this might have no beneficial effect since the strand is already completely solid.

* Technical contribution to the $45^{\text {th }}$ Steelmaking Seminar, May $25^{\text {th }}-28^{\text {th }}, 2014$, Porto Alegre, RS, Brazil. 

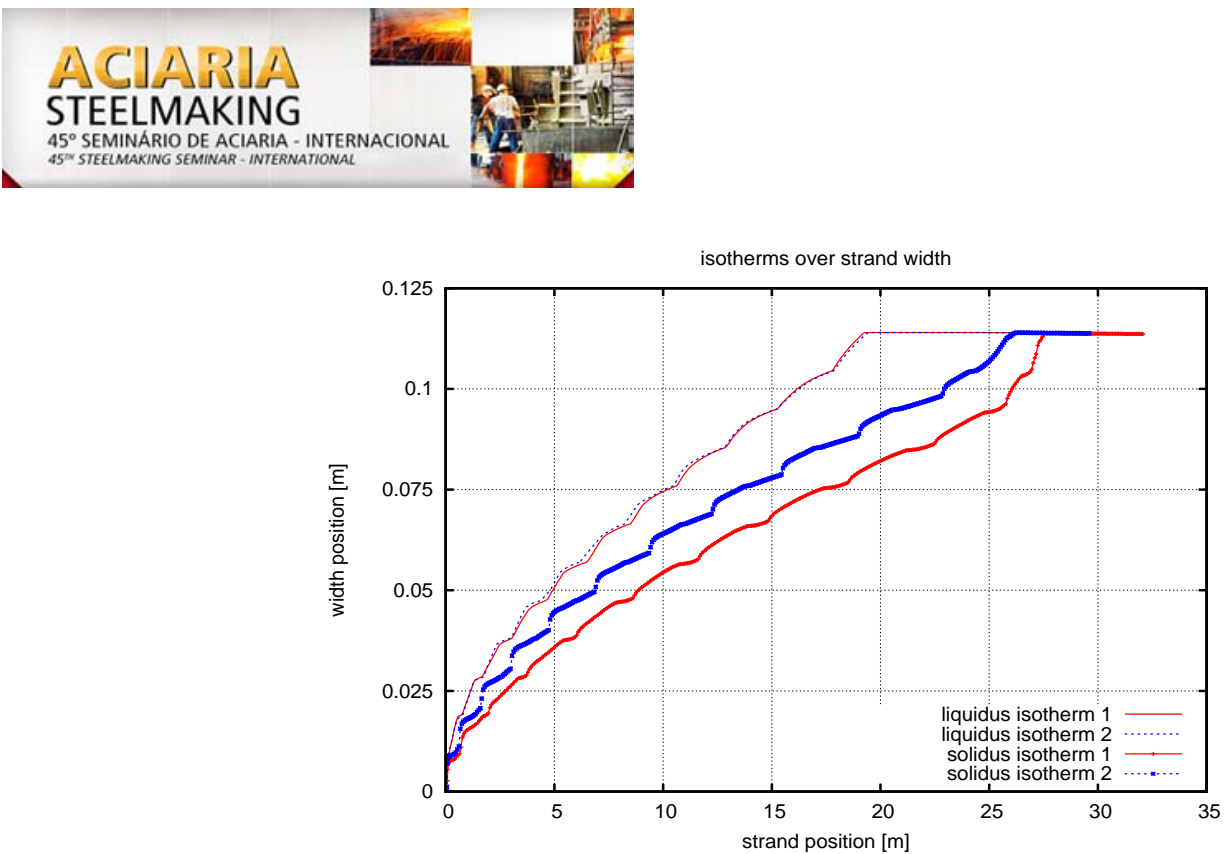

Figure 2. Liquidus isotherm and solidus isotherm of the narrow face at the center of the strand for the material parameters calculated with the DynaPhase database before the optimization (blue called isotherm 1) and after the optimization (red called 2). The liquidus isotherms are almost the same (dashed and solid lines) whereas large differences can be found for the solidus isotherms (dashed lines and solid lines with points). For the simulation with the improved database the solidus point is shifted downwards on the strand.

Simetal Dynacs 3D does not necessarily need DynaPhase. In case no DynaPhase is available Simetal Dynacs 3D uses pre-calculated material properties of so called grade groups. There are several sets of grade groups e.g. for carbon steel grades or stainless steel grades. Each group is valid for a certain concentration range whereas the material properties have been calculated for the mean concentrations of the group. All grade groups of one set together cover the usual scope of steel grades. For an actual steel grade Simetal Dynacs 3D will choose the grade group which is closest to its chemical analysis and will use the corresponding material properties to control the secondary cooling. The grid of grade groups is rather fine to keep the discrepancy between used grade group properties and actual steel grade properties small. However it is impossible to make such a grid very fine for all alloying elements. Otherwise one would end up with an enormous number of grade groups. One has to be aware of the fact that using Simetal Dynacs 3D without DynaPhase will usually lead to good results but is only an approximation.

The need for a precise determination of the final point of solidification is demonstrated on a billet caster. In the billet caster the soft reduction is not performed in segments which consist of several rolls, but it is performed by single pinch rolls. The final point of solidification shall correspond with the position of the rolls in order to prevent pipe formation, or center cracking, when soft reduction is done too early or after the strand is already solidified.

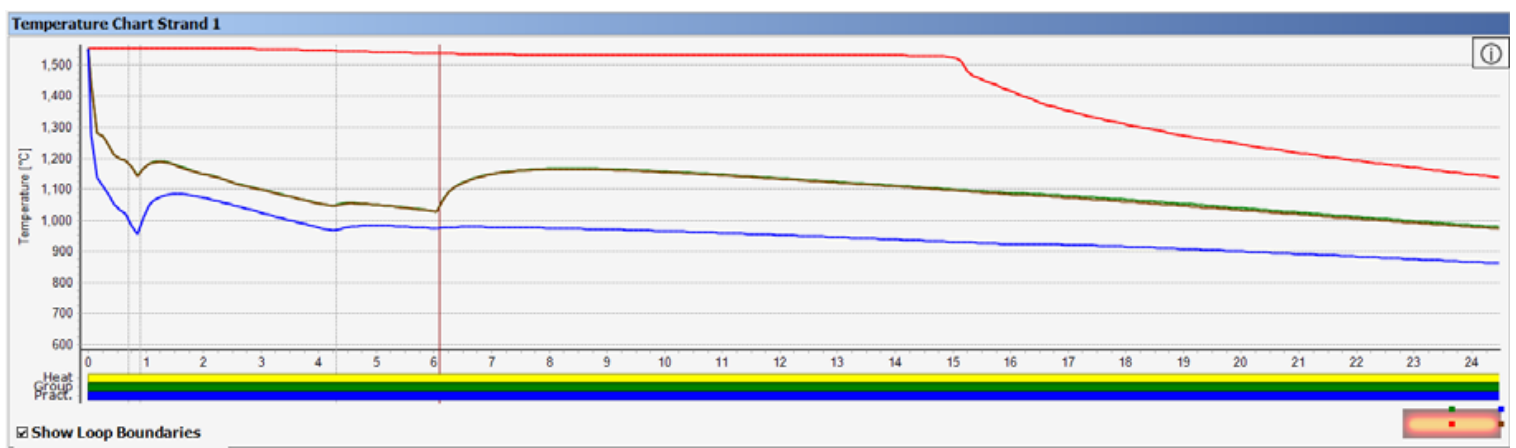

Figure 3. The temperature profile of the strand is calculated with Simetal Dynacs 3D. The final point of solidification is reached when the center temperature of the slab drops significantly.

* Technical contribution to the $45^{\text {th }}$ Steelmaking Seminar, May $25^{\text {th }}-28^{\text {th }}, 2014$, Porto Alegre, RS, Brazil. 
The temperature profile is calculated with Simetal Dynacs 3D and defines the 3 different regions of the center of the strand, liquid, mushy and completely solid regions are distinguished. The soft reduction is performed in the mushy zone. For relatively small alloying contents, like in low carbon steel grades or peritectic steel grades and lower casting speeds the mushy range is reduced. For the application of soft reduction in billet casting the mushy zone needs to be determined, so that the end of the mushy zone can be controlled to be at the position of one of the pinch rolls.

The temperature calculation is an important input for the calculation of the soft reduction range in DynaGap. The ideal soft reduction line is based on the materials input derived from the DynaPhase and the thermal calculation, which allow to calculate the range of the mushy zone. The determination of the final point of solidification is crucial to find the optimum setup for DynaGap.

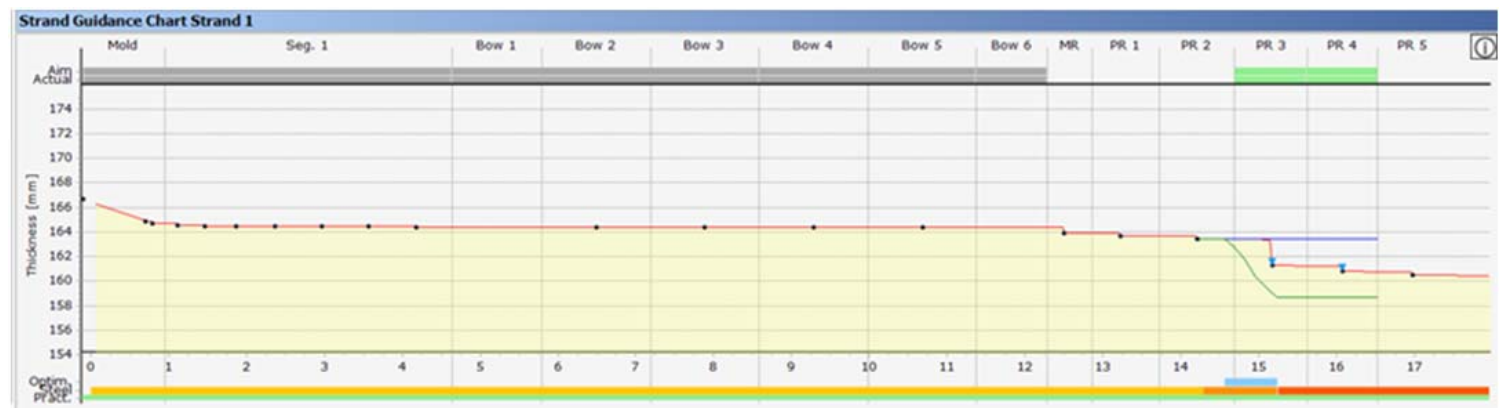

Figure 4. DynaGap calculates the roll gap based on the natural shrinkage of the strand which is overlapped by an additional reduction step performed in the mushy zone.

The solidus point is linked to the solidus temperature of the different steel grades. DynaPhase is in the position to calculate the solidus temperature very precisely. On site there is the possibility to operate the caster with a certain predefined steel grade groups, which is in many cases sufficiently accurate. In a specific situation it was necessary to describe the steel grades more precisely, so it has been found, that the solidus temperature varies in a range of $11^{\circ} \mathrm{C}$ for the peritectic steel grade group, which is accompanied by a shift of the solidus point of $20 \mathrm{~cm}$ (Table 1 ).

A shift of the solidus point by $20 \mathrm{~cm}$ is relatively small and might not have any major influence. Further analysis with Simetal Dynacs 3D revealed that $10 \mathrm{~cm}$ before the final point of solidification a mushy spot with $6 \mathrm{~mm}$ diameter is still present, which needs to be removed by means of soft reduction. The diameter of the pipe gives a hint about the amount of soft reduction.

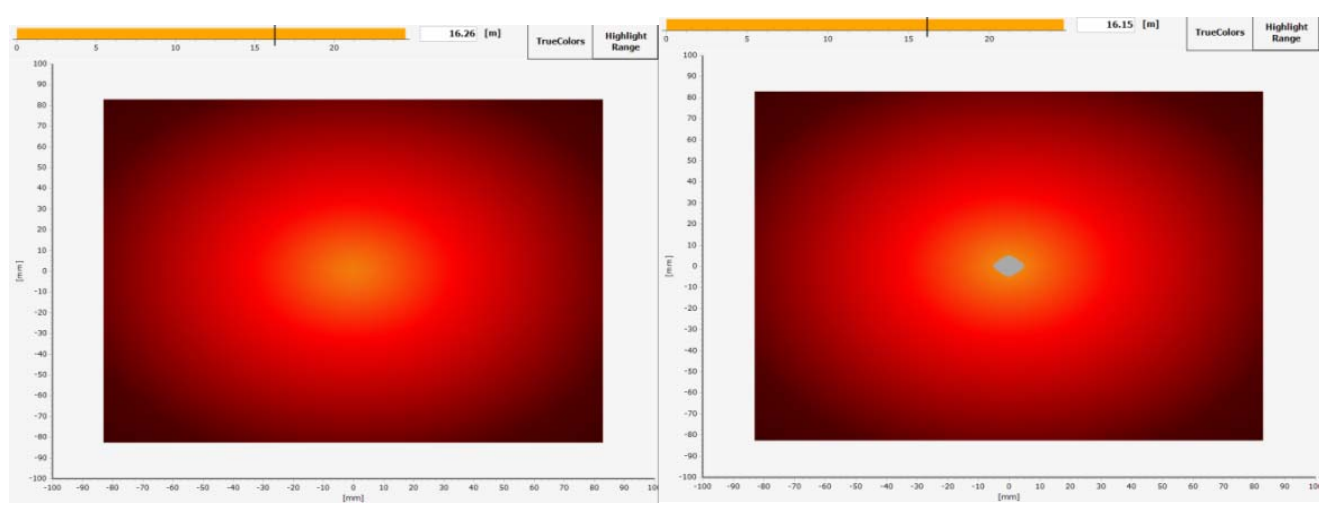

Figure 5. The final point of solidification for a certain caster, secondary cooling practice and casting speed is reached at $16.26 \mathrm{~m}$. About $10 \mathrm{~cm}$ before the final point of solidification a pipe containing liquid steel with a diameter of $6 \mathrm{~mm}$ is still present.

* Technical contribution to the $45^{\text {th }}$ Steelmaking Seminar, May $25^{\text {th }}-28^{\text {th }}, 2014$, Porto Alegre, RS, Brazil. 


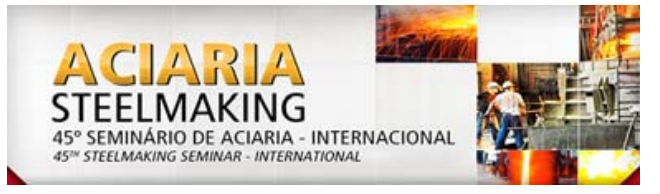

Table 1. Different representatives of the typical peritectic steel grade group (weight \%)

\begin{tabular}{ccccc}
$\begin{array}{l}\text { Different Steel grades } \\
\text { of the a typical } \\
\text { peritectic steel grade } \\
\text { group }\end{array}$ & $\begin{array}{c}\text { General } \\
\text { Steel Grade }\end{array}$ & $\begin{array}{c}\text { Client } \\
\text { specific } \\
\text { Steel Grade }\end{array}$ & $\begin{array}{c}\text { General } \\
\text { Steel Grade }\end{array}$ & $\begin{array}{c}\text { Client } \\
\text { specific } \\
\text { Steel Grade }\end{array}$ \\
\hline C & C014 & P235GH D & C016 & P295GH \\
\hline Mn & 0.14 & 0.14 & 0.16 & 0.16 \\
Si & 0.6 & 0.64 & 1 & 1.12 \\
$\mathbf{P}$ & 0.2 & 0.23 & 0.2 & 0.23 \\
$\mathbf{S}$ & 0.024 & 0.015 & 0.024 & 0.013 \\
$\mathbf{C r}$ & 0.01 & 0.009 & 0.01 & 0.008 \\
$\mathbf{N i}$ & 0.08 & 0.06 & 0.08 & 0.06 \\
$\mathbf{M o}$ & 0.08 & 0.1 & 0.08 & 0.1 \\
$\mathbf{C u}$ & 0.08 & 0.08 & 0.08 & 0.08 \\
$\mathbf{A l}$ & 0.08 & 0.28 & 0.08 & 0.28 \\
$\mathbf{N}$ & 0.04 & 0.035 & 0.04 & 0.045 \\
$\mathbf{T i}$ & 0.0048 & 0.01 & 0.0048 & 0.011 \\
$\mathbf{V}$ & 0.008 & 0.008 & 0.008 & 0.024 \\
$\mathbf{N b}$ & 0.008 & 0.008 & 0.008 & 0.015 \\
\hline & 0.008 & 0.05 & 0.008 & 0.015 \\
\hline Solidus Temp. [ ${ }^{\circ}$ C] & 1464 & 1462 & 1459 & 1453 \\
Solidus Point [m] & 16.25 & 16.05 & 16.05 & 16.15 \\
\hline Shift of the Solidus & & -0.2 & & +0.1 \\
Point [m] & & & & \\
\hline
\end{tabular}

The calculation with Simetal Dynacs 3D showed the ideal soft reduction amount, and the accurate position of the solidus point, which are essential for the operation of DynaGap.

Furthermore it has been shown, that the solidus temperature for different steel grades in the same steel grade group might differ by a value of $11^{\circ} \mathrm{C}$ which is followed by a shift of the solidus point of $20 \mathrm{~cm}$. A delayed reduction might result in undesired microstructures, with center cracking, a too early reduction might be followed by undesired pipe formation.

\subsection{DynaPhase Online}

So far we discussed how DynaPhase helps to control strand treatment technologies like soft reduction and therefore helps to improve the quality of the products. But DynaPhase online does more than that. It might happen, that the chemical analysis of a ladle is somewhat off the usual values. Since DynaPhase online uses the actual chemical analysis of the ladle for its calculation it informs the operator before start cast if the steel is peritectic. This might be out of the ordinary and since peritectic grades are more difficult to cast than non-peritectic grades the operator could adjust the casting practice accordingly. Fluctuations in the analysis influence the liquidus temperature which may result in a higher superheat than planned. Again, knowing this before casting the operator can try to optimize the casting practice for this altered situation.

Right now, we are working on the development of quality indices. This is a joint project with voestalpine Stahl, Linz, the department of metallurgy of the Mining University of Leoben and the research group of metallurgy of the School of Chemical Technology, Aalto University, Finland. With the actual chemical analysis indices

* Technical contribution to the $45^{\text {th }}$ Steelmaking Seminar, May $25^{\text {th }}-28^{\text {th }}, 2014$, Porto Alegre, RS, Brazil. 
between 0 and 1 are computed which indicate the risk of surface cracks and internal cracks. With the help of these indices the operator will be in the position to identify slabs with a high risk of surface defects which might need to be inspected or might need surface treatment.

\section{CONCLUSION}

We have described the thermodynamic software package DynaPhase in some details. Thereby we emphasized the importance of a good thermodynamic database for any prediction made by DynaPhase. Therefore we use new high precision DSC measurements to improve our database. So far we extended the range of validity for Si (up to $3.5 \mathrm{wt} \%$ ) and Al (up to $3.5 \mathrm{wt} \%$ ). Further experimental investigations are underway to further improve the database.

We demonstrated in two examples how the correct calculation of material properties with DynaPhase influences the accuracy of level 2 cooling control systems like Simetal Dynacs 3D. Accurate prediction of solid fractions is indispensable for effective use of strand treatment technologies as soft reduction. Accurate calculations of DynaPhase can also help to handle unusual fluctuations of the chemical analysis by adjusting the casting practice. This could prevent quality problems or at least could help to identify strands which might have some quality issues.

\section{Acknowledgement}

Financial support by the Austrian Federal Government (in particular from Bundesministerium für Verkehr, Innovation und Technologie and Bundesministerium für Wirtschaft, Familie und Jugend) represented by Österreichische Forschungsförderungsgesellschaft $\mathrm{mbH}$ and the Styrian and the Tyrolean Provincial Government, represented by Steirische Wirtschaftsförderungsgesellschaft $\mathrm{mbH}$ and Standortagentur Tirol, within the framework of the COMET Funding Programme is gratefully acknowledged.

\section{REFERENCES}

1 Mörwald K, Dittenberger K, Hohenbichler G. Improvement of secondary cooling control by on-line temperature calculation. In: ISS. $80^{\text {th }}$ Steelmaking Conference; 1997; Chicago, USA. ISS; 1997.

2 Stiftinger $\mathrm{M}$, Reichetseder $\mathrm{F}$, Resch $\mathrm{H}$, Eichinger $\mathrm{A}$. Design and dynamic control of secondary cooling systems for slab casters. AISE; 2002.

3 Chimani C, Watzinger J, Shan G, Resch H. Metallurgical strand treatment for direct hot charging. CCC, Linz; 2000.

4 Resch $\mathrm{H}$. Precipitation modeling: A tool for predicting cracks and quality. CCC, Linz; 2000.

5 Thalhammer M, Federspiel F, Mörwald K, Hödl H. Metallurgical, operational and economic benefits of VAI Smart/ASTC. AISE; 2000.

6 Ilie S, Fuchs R, Etzeldorfer E, Chimani C, Mörwald K. Slab quality improvement through soft reduction technology. CCC, Linz; 2008.

7 Morton J, Skrube S and Shen J. Application of DynaGap Soft Reduction ${ }^{\circledR}$ to high quality Blooms. AISTech; 2011.

8 Hauser K, Dittenberger K, Hahn S, Chimani C, Fürst C, Ilie S, Lindenberger S, et al. Dynamic 3D Heat transfer Simulation of Continuous Casting. ECC; 2008.

* Technical contribution to the $45^{\text {th }}$ Steelmaking Seminar, May $25^{\text {th }}-28^{\text {th }}, 2014$, Porto Alegre, RS, Brazil. 
9 Dittenberger K, Hahn S, Hauser K, Chimani C, Ilie S, Lindenberger L. Dynamic 3D heat transfer simulation of continuous casting. International Journal of Cast Metals Research. 2009;22(1-4):115-118.

10 Ramstorfer F, Dittenberger K, Hauser K, Hahn S. Dynacs 3D - The new dimension in secondary cooling for slab casters. In: ECCC - Electronic Colloquium on Computational Complexity; 2011, Düsseldorf, Germany.

11 Laitinen E, Neittaanmäki P. On numerical simulation of the continuous casting process. Journal of Engineering Mathematics; 1988;22:335-354.

12 Louhenkilpi S, Laitinen E, Nieminen R. Real-time simulation in continuous casting of steel. Metallurgical Transactions B. 1993;24B:685-693.

13 Camisani-Calzolari FR, Craig IK, Pistorius PC. Speed disturbance compensation in the secondary cooling zone in continuous casting. ISIJ International. 2000;40(5):469-477.

14 Miettinen J. Mathematical simulation of interdendritic solidification of low-Alloyed and stainless steels. Metall. Trans. A. 1992;23A:1150-70.

15 Miettinen J. Calculation of solidification-related thermophysical properties for steels. Metall. Trans. B. 1997;28B:281-97.

16 Miettinen J. Mathematical simulation of interdendritic solidification of lowalloyed and stainless steels [PhD thesis]. Espoo: Laboratory of Metallurgy, Helsinki University of Technology; 1992.

17 Hillert M. Hardenability concepts with application to steel. edited by D.V. Doane and J.S. Kirkaldy, Warrendale, PA, The Metallurgical Society of AIME, p. 5-27, 1978.

18 Presoly P, Pierer R, Bernhard C. Identification of defect prone peritectic steel grades by analyzing high temperature phase transformations. J. Metall. Trans. A. 2013;44A(12):5377-88.

19 Presoly P, Pierer R, Bernhard C. Linking up of HT-LSCM and DSC measurements to characterize phase diagrams of steels. In: IOP Conference Series (MCWASP XIII): Materials Science and Engineering; 2012; Schladming, Austria. 2012. n. 33012064.

20 FactSage [homepage]. Aachen: FactSage, 2014. Available at: http://www.factsage.com/

21 Thermo-Calc Software [homepage]. Stockholm: FactSage, 2014. Available at: http://www.thermocalc.com/.

* Technical contribution to the $45^{\text {th }}$ Steelmaking Seminar, May $25^{\text {th }}-28^{\text {th }}, 2014$, Porto Alegre, RS, Brazil. 